\title{
Infestation par Necator americanus de Souris blanches rendues immunotolérantes par des injections massives de protéines hétérologues
}

\author{
par Félix-A. LANCASTRE, Jean-Christian BAZIN, Yves-Jean GOLVAN, \\ Françoise-T. DELTOUR, Geneviève MOUGEOT et René HOUIN \\ et la collaboration technique de Josyane Bolognini et Chantal Dubois
}

(Laboratoire de Parasitologie du C.H.U. de Saint-Antoine 27, rue de Chaligny, 75 - Paris, $12^{\circ}$ )

\begin{abstract}
Résumé
Après avoir fait la revue des principales méthodes utilisées jusqu'ici pour briser la résistance à l'infestation d'animaux de laboratoire par des Nématodes, en particulier les Ancylostomidés, les auteurs décrivent une méthode provoquant une paralysie immunitaire chez la Souris blanche, et permettant l'accomplissement du cycle de Necator americanus.

Il s'agit d'injections massives et répétées de blanc d'œuf dans la cavité péritonéale. Au bout de 29 jours, des Vers adultes, deux mâles et quatre femelles, ont été récupérés chez deux Souris. Ces Vers étaient morphologiquement normaux et se nourrissaient de sang, mais n'ont pas pondu.

Pour les auteurs, la paralysie immunitaire induite chez la Souris permet de considérer comme relative la notion de spécificité parasitaire.
\end{abstract}

\section{Summary}

After a review of the principal methods permitting infection of laboratory animals by Nematodes, mostly Ancylostomidae, a new method inducing an immunotolerance state and allowing infection by Necator americanus, the human hookworm, is described by the authors.

The technique consists of massive and repeated injections with white of egg in peritoneal cavity of mice. Twenty nine days later, two males and four females worms, looking normal and ingesting blood, were recovered from two mice, but these worms were sterile.

These results induce the authors to consider as relative the notion of parasite specificity. 
Les études au laboratoire portant sur les Ancylostomidés humains (Ancylostoma duodenale et Necator americanus), et se proposant comme but certains aspects de leur physiologie, sont limitées par force aux formes larvaires libres, rhabditoïdes et strongyloïdes, obtenues par coproculture. Mais la partie purement parasitaire du cycle, comprenant la maturation sexuelle, se déroule dans le système vasculaire, l'arbre respiratoire et le tube digestif de l'homme. Ce fait rend très difficile toute expérimentation réelle sur ces deux vers, dont la physiologie est surtout connue grâce aux nécropsies et aux changements humoraux et tissulaires qu'entraîne leur présence.

Bien entendu, outre l'étude des Ancyclostomidés parasites d'autres mammifères, de nombreux auteurs ont tenté d'infester par $A$. duodenale et $N$. americanus divers animaux de laboratoire. Dès 1907, Ashford et King tentaient l'infestation percutanée d'un cobaye par des larves de $N$. americanus et constataient, trois jours après, à l'autopsie, une hémorragie pulmonaire et la présence de larves vivantes (in Schwartz et Alicata, 1934). Par la suite, Stiles (1922) aurait obtenu ( $\&$ apparently $)$ des adultes de $N$. americanus chez le chien. De même, Schwartz et Alicata (1934) ont étudié chez le cobaye les différents stades larvaires du même nématode dans les poumons et l'intestin, Nichols (1936) et Chin Thack Soh (1958), ayant infesté des souris par $A$. duodenale, $A$. caninum et $N$. americanus, ont établi avec précision la distribution des larves dans les différents tissus et organes ( visceral larva migrans $»$ ). Ces auteurs ont constaté une rapide disparition des larves des poumons, des parois et du contenu de l'intestin, au cours des deux premières semaines, même à la suite d'infestations massives. Citons encore Yoshida (1966), qui a observé dans les poumons et l'intestin de chiots infestés par $A$. duodenale et $N$. americanus des larves restées au stade III, et Yoshida et Fukutome (1967) qui, ayant infesté des lapins, constatèrent la présence d'œufs fertiles dans l'utérus de $N$. americanus femelles, entre le $60^{\circ}$ et le $63^{\circ}$ jour. Enfin, Sen et Seth (1967) ont obtenu chez de jeunes hamsters un «développement complet », avec formation d'adultes immatures $\left(21^{\circ}-27^{\circ}\right.$ jour $)$ et d'adultes achevés au $35^{\circ}$ jour. Dans les fèces d'un des hamsters, les auteurs ont trouvé des œufs fertiles qui ont donné des larves en coproculture.

En 1929, Sandground, faisant la revue des travaux sur la spécificité parasitaire chez les Helminthes et autres Métazoaires, insiste sur deux facteurs qu'il estime très importants : 1) la résistance due à l'âge — déjà observée par Looss (1911); 2) la résistance acquise, analogue à une prémunition.

Dans l'un et l'autre cas, il faut se référer à l'hôte. C'est lui le plus important. En. effet, écrit Sandground : «Specifity..., is a reciprocical relationship between two organisms, and the phenomenon must also be investigated from the host aspect. Therefore, let us now turn to consider any changes that may occur in the course of the development of the host. What essentially happens in connection with age resistance is that the host, after reaching a certain age, develops characteristics that render it immune to particular parasites ».

Cette influence prépondérante de l'âge a été bien mise en évidence par les auteurs que nous avons cités plus haut et qui ont obtenu un développement des Ancylostomidés 
humains chez de jeunes animaux, sans pouvoir toujours poursuivre au-delà du stade larvaire III. Il s'y ajoute l'immunité plus ou moins efficace acquise lors d'une primoinfestation, qui, même avortée, peut renforcer les mécanismes de rejet survenant au cours d'infestations ultérieures.

De nombreux chercheurs, travaillant sur les Nématodes, ont tenté de briser cette double résistance, induite par l'âge de l'hôte et (ou) les infestations antérieures. Certains ont utilisé les hormones sexuelles: Haley sur Nippostrongylus muris (1954, 1958), Clapham sur Syngamus trachea (1939), Ackert et Dewhirst (1950), Todd et Crowdus (1951), Todd et Hollingsworth (1952), sur Ascaridia galli, Stoll, sur Haemonchus contortus (1940), Mathies, sur l'oxyure de la souris, Aspiculuris tetraptera (1954). D'autres ont utilisé la suppression de la réponse inflammatoire cellulaire provoquée par la cortisone: Galliard et Berdonneau, sur Strongyloïdes stercoralis (1953), Roman, sur Strongylö̈des ratti (1956), Briggs (1959) et Olson (1959), sur Litomoïdes carinii, Cross Jr., sur Nematospirö̈des dubius (1960), Bezubik, sur Strongylö̈des papillosus (1960), Ritterson, sur Trichinella spiralis (1960), Parker, sur Nippostrongylus brasiliensis (1961).

En 1966, Miller, ayant traité de jeunes chiens par la méthyl-prednisolone, réussit à les infester par $N$. americanus et à obtenir des adultes mâles et femelles, sexuellement mûrs, au bout de 42 jours.

$\mathrm{Si}$ on ignore encore tout du mécanisme d'action des hormones sexuelles (d'autant plus qu'il existe des effets contradictoires), on pense généralement que c'est la suppression de la réponse inflammatoire chez les animaux traités par la cortisone qui permet l'établissement et le maintien de parasites inhabituels.

La plupart des vers cités plus haut accomplissent leur cycle à travers l'organisme et finalement vont se fixer, plus ou moins profondément, dans la muqueuse de l'intestin ou de l'arbre respiratoire (Syngamus trachea). Ils sont au contact des cellules ou du sang, parfois s'en nourrissent. Il est donc nécessaire que les organes opposent le minimum de réactions défensives contre cette intrusion. Les hormones sexuelles et les hormones corticoïdes provoqueraient une sorte de retour à un état juvénile de l'organisme tout entier, au point de vue immunologique, un état de grande tolérance immunitaire.

On peut considérer les parasites comme des sortes de greffons habituellement bien adaptés à leurs hôtes. Dans ce cas, il est évident que la capacité d'adoption de l'hôte est le facteur sine qua non à considérer pour que prenne cette «greffe». Un état d'immunotolérance est donc nécessaire.

Nous avons tenté de parvenir à cet état d'immunotolérance par une autre méthode en prenant pour point de départ les travaux de Dubert et Paraf (1957), Liacopoulos et coll. (1961 et 1962), Halpern et coll. (1966). Ces auteurs ont réussi à provoquer chez le cobaye et le lapin, au moyen d'injections massives et répétées de protéines hétérologues, telles la sérum-albumine et les globulines bovines et humaines, un état de paralysie immunitaire inhibant l'hypersensibilité de type retardé et allongeant la durée de survie d'homogreffes cutanées. 
Nous avons mis au point une méthode simple d'adaptation de parasites à des hôtes inhabituels, en injectant des doses massives d'un antigène protéinique banal comme le blanc d'œuf de poule à des animaux de laboratoire. Cette méthode a été appliquée dans le cas d'infestation de souris blanches par $N$. americanus.

\section{Protocole expérimental}

\section{Matériel.}

Un lot de 20 souris, adultes, des deux sexes, reçoit pendant 10 jours une injection intrapéritonéale de $2 \mathrm{ml}$ de blanc d'œuf. Arrêt le $11^{\circ}$ jour. Infestation le $12^{\circ}$ : injection sous-cutanée de 300-500 larves strongylö̈des infestantes de $N$. americanus recueillies par la méthode de Baermann à partir de coprocultures de 7-10 jours. Ces coprocultures sont faites avec des selles de sujets originaires des Antilles, reconnus infestés à la consultation de Pathologie parasitaire et tropicale de l'Hôpital Saint-Antoine. Dès le lendemain de l'infestation, et tous les deux jours, les souris reçoivent une injection de $2 \mathrm{ml}$ de blanc d'œuf et cela jusqu'au terme de l'expérience.

\section{Résultats.}

De nombreuses souris sont mortes au cours des deux premières semaines suivant l'infestation. L'autopsie a montré des lésions hémorragiques au niveau des poumons où des larves III ont été recueillies $\left(7^{\circ}-12^{\circ}\right.$ jour $)$.

\section{Tableau I}

\begin{tabular}{||c|c|l|}
\hline \multicolumn{1}{|c|}{ Date } & Localisation & \multicolumn{1}{c|}{ Observations } \\
\cline { 1 - 3 } $7^{\mathrm{e}}-12^{\mathrm{e}}$ jour $\ldots \ldots \ldots \ldots$ & Poumons & $\begin{array}{l}\text { Nombreuses larves III } \\
\text { Pas de différenciation } \\
\text { sexuelle. }\end{array}$ \\
\hline $12^{\mathrm{e}}-21^{\circ}$ jour $\ldots \ldots \ldots \ldots$ & Duodénum & $\begin{array}{l}\text { Larves III sans différencia- } \\
\text { tion sexuelle. } \\
\text { Larves IV sexuellement dif- } \\
\text { férenciées. }\end{array}$ \\
\hline
\end{tabular}

Sur les 7 souris ayant survécu au-delà, trois sont mortes sans qu'une autopsie ait pu être pratiquée, deux ont été sacrifiées le $21^{\circ}$ jour, et les deux dernières le $29^{\circ}$ jour de l'infestation.

Chez toutes les souris mortes, les poumons, la trachée et l'œsophage étaient recueillis ensemble et dilacérés dans une boîte de Pétri contenant du sérum physio- 
logique tiède. L'intestin grêle était coupé dans toute la longueur et également examiné dans du sérum physiologique.

Les larves III ont été trouvées au niveau des poumons entre le $7^{\circ}$ et le $12^{\circ}$ jour, et de l'intestin le $12^{\circ}$ et le $21^{\circ}$ (tableau I). Les larves IV ont été trouvées, fixées à la muqueuse du duodénum inférieur le $21^{\circ}$ jour (tableaux I et II). Au $29^{\circ}$ jour, il a été trouvé dans l'intestin des deux dernières souris des individus manifestement adultes, deux mâles et quatre femelles (tableau III). Ces vers se maintenaient en place, un morceau de muqueuse engagé dans leur capsule buccale.

A aucun moment nous n'avons trouvé d'œufs dans les crottes des souris.

\section{Tableau II}

CARACTÉRISTIQUES MORPHOLOGIQUES DES $2 \mathrm{~N}$. americanus (femelles immatures) recueillies au $21^{\circ}$ jour

\begin{tabular}{|c|c|c|}
\hline \multirow{2}{*}{$\begin{array}{c}\text { Caractéristiques } \\
\text { (dimensions en } \mathrm{mm} \text { ) }\end{array}$} & \multicolumn{2}{|c|}{ Stade IV } \\
\hline & $\Varangle \mathrm{n}^{\circ} 1$ & $q n^{\circ} 2$ \\
\hline Longueur totale $\ldots \ldots \ldots \ldots \ldots \ldots$ & 3,230 & 3,750 \\
\hline Largeur maximum ............. & 0,180 & 0,230 \\
\hline Longueur de l'œsophage..$\ldots \ldots \ldots \ldots$ & 0,700 & 0,765 \\
\hline $\begin{array}{l}\text { Distance de l'extrémité antérieure à } \\
\text { l'anneau nerveux } \ldots \ldots \ldots \ldots \ldots \ldots \ldots\end{array}$ & 0,330 & 0,360 \\
\hline $\begin{array}{l}\text { Distance de l'extrémité antérieure à la } \\
\text { vulve } \ldots \ldots \ldots \ldots \ldots \ldots \ldots \ldots \ldots\end{array}$ & 1,620 & 1,965 \\
\hline Longueur de la queue...$\ldots \ldots \ldots \ldots$ & 0,180 & 0,780 \\
\hline
\end{tabular}

\section{Infestation de souris témoins.}

Parallèlement, des souris non traitées ont été infestées. Les autopsies pratiquées à partir du $2^{\circ}$ jour ont révélé la présence de larves III, en petit nombre, dans les poumons, jusqu'au $8^{\circ}$ jour suivant l'infestation. Nous n'avons pas retrouvé de larves III dans l'intestin de ces souris, ni, bien entendu, de larves IV, ni de $N$. americanus adultes. Il y a eu très probablement destruction et phagocytose des larves III au niveau des poumons, ce qui concorde avec les résultats obtenus par Schwartz et Alicata (1934), Nichols (1956), Chin Thack Soh (1958).

\section{Discussion}

D'après Chandler et Read (1966), les dimensions de $N$. americanus adulte sont de 10-11 mm pour la femelle (largeur: $0,4 \mathrm{~mm}$ ), et $7-8 \mathrm{~mm}$ pour le mâle (largeur: $0,3 \mathrm{~mm}$ ). Les spécimens les plus grands que nous ayons recueillis mesurent $6,42 \mathrm{~mm}$ (\$) et $6,00 \mathrm{~mm}\left(\sigma^{*}\right)$, soit respectivement $40 \%$ et $15 \%$ de moins que les vers normaux, 
« humains ». Mais ils étaient vivants, fortement ancrés dans la muqueuse duodénale, et colorés en rose par le sang ingéré.

\section{Tableau III}

CARACTÉRISTIQUES MORPHOLOGIQUES DES $N$. americanus

( 2 mâles, 4 femelles) recueillis le $29^{\circ}$ jour

\begin{tabular}{|c|c|c|c|c|c|c|}
\hline $\begin{array}{c}\text { Caractéristiques } \\
\text { (dimensions en } \mathrm{mm} \text { ) }\end{array}$ & $\begin{array}{l}\text { Ver } \\
\mathrm{n}^{\circ} 1\end{array}$ & $\begin{array}{l}\text { Ver } \\
\mathrm{n}^{\circ} 2\end{array}$ & $\begin{array}{l}\text { Ver } \\
\mathrm{n}^{\circ} 3\end{array}$ & $\begin{array}{l}\text { Ver } \\
\mathrm{n}^{\circ} 4\end{array}$ & $\begin{array}{l}\text { Ver } \\
\mathrm{n}^{\circ} 5\end{array}$ & $\begin{array}{l}\text { Ver } \\
\mathrm{n}^{\circ} 6\end{array}$ \\
\hline Sexe...$\ldots \ldots$ & $0^{*}$ & $q$ & $q$ & $q$ & $q$ & $\delta$ \\
\hline Longueur totale. & 4,880 & 6,420 & 5,700 & 4,700 & 4,310 & 6,000 \\
\hline Largeur maximum & 0,260 & 0,300 & 0,270 & 0,230 & 0,180 & 0,260 \\
\hline $\begin{array}{l}\text { Longueur de l'œ- } \\
\text { sophage } \ldots \ldots \ldots \ldots\end{array}$ & 0,765 & 0,765 & 0,735 & 0,735 & 0,630 & 0,810 \\
\hline $\begin{array}{l}\text { Distance de l'ex- } \\
\text { trémité antérieure } \\
\text { à l'anneau ner- }\end{array}$ & & & & & & \\
\hline $\begin{array}{l}\text { veux ............ } \\
\text { Distance de l'extré- } \\
\text { mité antérieure à }\end{array}$ & 0,315 & 0,360 & 0,300 & 0,240 & 0,315 & 0,375 \\
\hline la vulve ......... & - & 3,330 & - & 2,880 & 2,300 & - \\
\hline $\begin{array}{l}\text { Longueur de la } \\
\text { queue } \ldots \ldots \ldots \ldots\end{array}$ & - & 0,780 & 0,230 & 0,170 & 0,270 & - \\
\hline
\end{tabular}

Ce résultat est supérieur à ceux obtenus par Ashford et King (1907), Nichols (1956), Chin Thack Soh (1958), et Yoshida (1966) qui, au mieux, récupérèrent dans l'intestin des animaux d'expérience des larves du $3^{\circ}$ stade (au $6^{\circ}$ jour pour Chin Thack Soh), du $4^{\circ}$ stade (au $22^{\circ}$ jour pour Yoshida).

Schwartz et Alicata (1934) ont trouvé des larves du $4^{e}$ stade entre le $9^{\circ}$ et le $16^{\circ}$ jour dans l'intestin de cobayes (taille maxima; $\uparrow 1,943 \mathrm{~mm} ;$ đ̊ $1,855 \mathrm{~mm}$ ). La plupart des expérimentations chez ces auteurs ont abouti à la réalisation de «larva migrans » viscérale.

Miller (1966), Yoshida et Fukutome (1967), et Sen et Seth (1967), sont les seuls auteurs que nous connaissons à avoir obtenu le développement complet de $N$. americanus chez l'animal d'expérience, jusqu'à la maturation sexuelle, bien que les œufs obtenus dans l'expérimentation de Miller n'aient pu s'embryonner. Du fait de la mortalité élevée chez nos souris traitées (65\% pendant les deux premières semaines de l'infestation), il ne nous a pas été permis jusqu'à présent de mener l'expérience jusqu'à la formation et la ponte d'œufs. L'adjonction d'antibiotiques à la nourriture ou au blanc d'œuf injecté abaisse fortement ce taux de mortalité et doit nous permettre d'obtenir bientôt des $N$. americanus fertiles. Il serait alors possible d'accomplir le cycle complet de ce Nématode uniquement sur la souris blanche. 
Les résultats que nous avons obtenus peuvent s'expliquer par le blocage de la réaction immunologique primaire et l'arrêt de la production d'anticorps, ce qui permet au ver d'accomplir son développement dans le sang, le poumon, la trachée, l'œsophage, et enfin le duodénum de la souris, et de se nourrir sans dommage du plasma, des globules sanguins et des cellules épithéliales d'un hôte absolument inhabituel. Il y a indiscutablement une augmentation de la susceptibilité des souris à l'infestation par $N$. americanus, bien que le rendement soit bas, et que 0,5 à $1 \%$ seulement des larves infestantes parviennent au stade IV, et au-delà, dans notre expérimentation. Le mode d'action de l'immuno-inhibition induite par les protéines du blanc d'œuf chez la souris serait une compétition lors de la phase de reconnaissance des antigènes, par les cellules compétentes (Stiffel et coll., 1966). La durée et l'importance de l'inhibition étant proportionnelles à la quantité de protéine hétérologue administrée, on aura d'autant plus de chances d'obtenir des stades avancés de $N$. americanus que les injections se seront poursuivies plus longtemps, ce qui a été vérifié au cours d'expériences précédentes.

Ainsi, on peut penser que l'acceptation ou le rejet de $N$. americanus par un organisme est un mécanisme qui met en jeu l'immunité de type cellulaire, c'est-à-dire l'action des cellules immunologiquement compétentes (lymphocytes, peut-être macrophages). Toute action inhibant cette immunité permettra donc le développement de parasites inhabituels. Nous avons pu ainsi réaliser l'infestation de souris blanches par les formes hématophages de Entamoeba histolytica (Lancastre et coll., 1968) et, toujours chez la souris, obtenir la maturation sexuelle de Trichinella spiralis dans la cavité abdominale (Bazin et coll., 1969).

Nous sommes donc amenés à penser - et ici nous paraphrasons Sandground (1929) - que la spécificité est un phénomène qui doit être envisagé surtout du point de vue de l'hôte définitif, et que dans les relations hôte-parasite, la part de l'hôte peut être la plus importante.

A un certain niveau d'inertie immunologique, la notion de spécificité parasitaire considérée lato sensu tend à s'abolir. Telles sont les conclusions que nous pouvons tirer de cette expérimentation, et des travaux à paraître ou en cours semblent les confirmer.

\section{Bibliographie}

ACKert (J. E.) et Dewhirst (L. W.), 1950. - Resistance of fowls to parasitism affected by female sex hormone. J. Parasit., 36, Sect. 2 (Suppl.), 16.

Bazin (J.-C.), Lancastre (F.-A.), Le Fichoux (Y.), Mougeot (G.) (à paraître). - Trichinose expérimentale ( $2^{\circ}$ note). Annales Parasit. hum. comp. (Paris).

BEZUBIK (B.), 1960. - Effect of cortisone on the susceptibility of hamsters and guinea pigs to the Sheep and Rabbit strains of Strongyloides papillosus. J. Parasit., 46 (Suppl.), 30-31. 
BRIGGS (N. T.), 1959. - The effect of cortisone on natural resistance and acquired responses of the white Rat to infection with Litomosoides carinii. J. Parasit., 45 (suppl.), sect. 2, 37.

Chander (A. C.) et Read (C. P.), 1966. - Introduction to Parasitology, 10th edition, 822 pp., John Wiley and Sons, New-York.

CHIn Тнаск Sон, 1958. - The distribution and persistence of hookworm larvae in the tissues of mice in relation to species and to routes of inoculation. J. Parasit., 44, 515-519.

Clapham (P. A.), 1939. - On a sex difference in the infection rate of birds with Syngamus trachea. J. Helminth., 17, 192-194.

Cross Jr (J. H.), 1960. - The natural resistance of the white Rat to Nematospiroides dubius and the effect of cortisone on this resistance. J. Parasit., 46, 175-185.

Dubert (J.-M.) et Paraf (A.), 1957. - Etude quantitative des conditions nécessaires à l'apparition d'un état de tolérance immunitaire. C.R. Acad. Sciences (Paris), 244 686-688.

Galliard (H.) et Berdonneau (R.), 1953. - Strongyloidose expérimentale chez le Chien Effet de la cortisone. Résultats du test de Thorn à l'hormone corticotrope (A.C.T.H.). Annales Parasit., 28, 163-171.

Haley (A. J.), 1954. - A difference in the susceptibility of the male and female Hamster to infection with the Rat nematode, Nippostrongylus muris. J. Parasit., 40 (Suppl.), 42.

Haley (A. J.), 1958. - Sex difference in the resistance of Hamsters to infection with the Rat nematode, Nippostrongylus muris. Exptl. Parasit., 7, 338-348.

Halpern (B.-N.), Liacopoulos (P.), Martial-Lasfargues (C.) et Aractingi (R.), 1963. Allongement de la durée de survie des homo-greffes cutanées chez le Lapin et le Cobaye au cours de la paralysie immunitaire induite par l'administration de globulines hétérologues. C.R. Soc. Biologie (Paris), 157, 740-743.

Lancastre (F.-A.), Bazin (J.-C.), Gargouri (M.), Le Fichoux (Y.), Mougeot (G.), 1968. Amibiase expérimentale. Annales Parasit. hum. comp. (Paris), 43, 623-628.

Lincopoulos (P.), 1961. - Inhibition des réponses immunologiques après administration de doses élevées d’une protéine hétérologue. C.R. Acad. Sciences (Paris), 253, 751-753.

Liacopoulos (P.), Neveu (T.), Biozzi (G.) et Halpern (B.), 1962. - Inhibition de lhypersensibilité du type retardé au cours de la tolérance immunitaire non spécifique du Cobaye adulte. C.R. Acad. Sciences (Paris), 254, 3765-3767.

Mathies (A. W.), 1954. - The influence of sex on Mouse pin-worm infection. J. Parasit., 40, 702 .

Miller (T. A.), 1966. - The effect of a synthetic corticosteroid upon the susceptibility of Dogs to infection with the human hookworm, Necator americanus. J. Parasit., 52, 285-290.

Nichols (R. L.), 1956. - The etiology of visceral larval migrans. II. Comparative larval morphology of Ascaris lumbricoides, Necator americanus, Strongyloides stercoralis and Ancylostoma caninum. J. Parasit., 42, 363-399.

OLson (L. J.), 1959. - The cellular response of white Rats to Litomosoides carinni larvae as influenced by cortisone, age and previous infection. J. Parasit., 45, 519-532. 
PARKeR (J. C.), 1960. - Effect of cortisone on the resistance of the Guinea pig to infection with the Rat nematode Nippostrongylus brasiliensis. Exptl. Parasit., 11, 380-390.

RitTERSON (A. L.), 1960. - Attempts to modify innate resistance of Chinese Hamsters to Trichinella spiralis. J. Parasit., 46 (Suppl.), Sect. 2, 22.

Roman (E.), 1956. - Spécificité parasitaire de Strongyloides ratti, du Surmulot. Effets de la cortisone sur l'infestation d'autres Rongeurs par ce nématode. Annales Parasit., 31, $552-571$.

SAdUN (E. H.), 1951. - Gonadal hormones in experimental Ascaridia galli infection in Chickens. Exptl. Parasit., 1, 70-82.

SANDGRound (J. H.), 1929. - A consideration of the relation of the host specificity of helminths and other metazoan parasites to the phenomena of age resistance and acquired immunity. Parasitology, 21, 227-255.

Schwartz (B.) et Alicata (J. E.), 1934. - Development of the human hookworm, Necator americanus, in Guinea-pigs. Am. j. Hygiene, 20, 317-328.

SEN (H. G.) et SETH (D.), 1967. - Complete development of the human hookworm, Necator americanus in golden Hamsters, Mesocricetus auratus. Nature, 214, 6 mai, 609-610.

Stiffel (C.), Ben-Effraim (S.), Perramant (M.-F.), Liacopoulos (P.), 1966. - Compétition des antigènes. Etudes sur son mécanisme chez le Cobaye. Ann. Inst. Pasteur (Paris), $111, \mathrm{n}^{\circ} 5$ (suppl.), 94-109.

StILes (C. W.), 1922. - Necator americanus in the Dog. (In Minutes Helm. Soc. Washington). J. Parasit., 8, 95.

Stoll (N. R.), 1940. - Worm host systems as labile mechanisms : a view of the nematodeRuminant problem. J. Am. Vet. Assoc., 96, 305-308.

Todd (A. C.) et Crowdus (D. H.), 1951. - Methyl testosterone in the diet of Chicks and growth of the nematode Ascaridia galli. J. Parasit., 37, 322.

Todd (A. C.) et Hollingsworth (K. P.), 1952. - Host sex as a factor in development of Ascaridia galli. Exptl. Parasit., 1, 303-308.

Yoshida (Y.), 1966. - Morphological differences between Ancylostoma duodenale and Necator americanus in the fourth larval stage. J. Parasit., 52, 122-126.

Yoshida (Y.) et Fukutome (S.), 1967. - Experimental infection of Rabbits with the human hookworm, Necator americanus. J. Parasit., 53, 1067-1073. 